\title{
Critical Review of Methods for Sampling, Analysis, and Monitoring of Vapor-Phase Toluene Diisocyanate
}

\author{
Steven P. Levine \\ Department of Environmental Health Sciences, School of Public Health, University of Michigan, \\ Ann Arbor, Michigan
}

This article is a critical review of some of the methods that have been used for the sampling, analysis, and monitoring of vapor-phase toluene diisocyanate (TDI). Only some of the methods that have received relatively widespread application have been addressed in this article. This review includes a "tutorial style" discussion of basic definitions and basic principles and procedures of quality control and metrology for sampling, analysis, and monitoring. One critical issue that is addressed: Are there methods and monitoring instruments available that are capable of sampling and analyzing or monitoring TDI vapor with sufficient speed and sensitivity to satisfy the requirements of the current ACGIH $^{\circledR}$ threshold limit values (TLVs ${ }^{\circledR}$ ) for TDI vapor?

Keywords Toluene Diisocyanate, TDI, Quality Assurance, Exposure Assessment, Monitoring, Air Sampling, Metrology

This article is a critical review of some of the methods that have been used for the sampling, analysis, and monitoring of toluene diisocyanate (TDI) vapor. Other publications have addressed some of these same issues. ${ }^{(1-8)}$ Not all sampling, analysis, and monitoring methods that have been developed have been addressed in this article. The only methods that are addressed are those

1. that have received relatively widespread application,

2. for which there is important, new information that has not heretofore been widely available, and

3. that are centrally important to answering the question: "Are methods available that are capable of monitoring TDI vapor with sufficient speed and sensitivity to satisfy the requirements of the current $\mathrm{ACGIH}^{\circledR} \mathrm{TLVs}^{\circledR}$ for TDI vapor?"

Proper exposure assessment is critical in the risk analysis process, since both exposure and effect measurements are required. Without proper exposure assessment, studies of health effects cannot be used in a quantitative strategy to establish permissi- ble exposure limits or TLVs ${ }^{\circledR} .{ }^{(9-13)}$ Furthermore, TLV $^{\circledR}{ }^{\circledR}$ should not be set based on perceived methodological limitations. Rather, methodology must be available to serve the process of setting TLVs ${ }^{\mathbb{R}}$ based on causally related exposure-health effects data. This review has been performed without any attempt to link methodological inadequacies to the strengths or weaknesses of specific health effects studies. That has been done previously. ${ }^{(11,12)}$ As in any scientific endeavor, the state-of-theart sampling, analysis, and monitoring for TDI is an evolutionary process. Clearly, newer methods replace older techniques as our knowledge expands.

This review includes a "tutorial style" discussion of basic definitions and basic principles and procedures of quality control and metrology for sampling, analysis, and monitoring. These considerations may seem to be too basic, or even peripheral to the assessment of TDI exposure. However, those principles and procedures are the primary measures of whether or not an analytical method can be considered to be quantitative.

\section{DEFINITIONS OF TERMS}

Any evaluation of data regarding the health effects of TDI vapor must start with an evaluation of the methods used for sampling, analysis, and monitoring.

Isocyanates: Isocyanates can be divided into several classes or types depending upon their structure. In industry, TDI is most frequently used as a mixture of 2,4 and 2,6 isomers. Not addressed in this article are otherisocyanate compounds, isocyanatecoated aerosols, prepolymers of TDI synthesized by pre-reacting some or all of their - NCO groups with a polyol (molecules containing more than one $-\mathrm{OH}$ group), and the total reactive isocyanate groups (TRIGs) resulting from the monomers and the polymeric, prepolymeric, or partially reacted isocyanate materials.

Vapors and Aerosols: Airborne TDI monomer exists primarily as a vapor. The polymeric, prepolymeric, modified, or partially polymerized isocyanates will exist primarily as an aerosol, not vapor, due to their higher molecular weight. This aerosol may or may not be amenable to sampling, analysis, or monitoring by the same techniques as free TDI monomer can. 
Quality Assurance Principles: All sampling, analysis, and monitoring methods must be used with specific quality assurance and control (QA/QC) procedures. Specifications for use must be detailed for calibration curves; limits of detection (LOD) and quantitation (LOQ); field and laboratory spikes, blanks, and duplicates; and analysis of data using Shewhart quality control charts.

It is important to remember, when evaluating exposure data at low concentrations, what the differences are in reporting requirements for isocyanates present at concentrations near the detection limit. For results below the LOD, the report must state "ND" on the data table. ND, or " $<$ LOD" must be defined as "nondetectable = (value)" for each case. Sometimes, ND is considered to be equal to zero, which is unwarranted. For results at concentrations between the LOD and LOQ, the report may state a numerical value with an appropriate footnote to that number, or " $<\mathrm{LOQ}$," and the LOQ value must be given. Numerical results without the "<LOQ" notation attached are not warranted in this concentration domain. If LOD and LOQ are undefined, the reliability of data is questionable at low concentrations. It is an unusual publication in which those specifications are actually given. When these specifications are missing, such data may be indefensible.

The quality of the information resulting from any study depends on knowing what quality control procedures were used to generate the data. This is not to say that a small bias or source of variance will be introduced without suitable documentation and publication of the quality control information. It is to say that the data may be difficult to interpret because the source and extent of variance will be unknown, and thus the information derived from the data may be wrong.

Instrument Metrology: An important issue is "How does one evaluate results that are obtained when using a direct reading instrument?" The American Industrial Hygiene Association (AIHA) published a paper that defines an official position on metrological requirements for air monitoring instruments. ${ }^{(14)}$ These requirements include calibration, accuracy, selectivity, specificity, detection limits, ranges, and response times. This article, and the requirements detailed therein, are still current and applicable to all air monitoring instruments. This AIHA document also requires knowledge of the conditions under which a direct reading instrument should be evaluated. Metrological specifications are very important in the case of isocyanates. Even in the laboratory, subtle changes in test specifications may strongly affect results.

Caution must be used when extrapolating results obtained during laboratory evaluation of instruments to the situation that will be encountered in an actual workplace. This is not to say that the extrapolation should not be done, but simply that due care should be exercised.

Subtle issues of instrument design may have a significant and not easily measured effect on the validity of laboratory evaluation studies. Hallmarks of sound exposure evaluation data obtained when using good quality monitoring instruments requires attention to the detailed specifications of good metrological practice and conservative extrapolation of laboratory evaluation studies to field practice. The literature must be carefully examined in cases where direct reading instruments have been used for TDI vapor. In cases in which instruments have been used where requisite information is lacking, the exposure assessment data must be considered in light of the missing metrological information. ${ }^{(12)}$

\section{SPECIFIC AIR SAMPLING AND ANALYSIS METHODS}

Criteria: For the purpose of this article, air sampling and analysis methods for TDI vapor have been divided into two tiers:

- Tier One methods are those that have been reviewed in this article in some detail.

- Tier Two is the category in which all other methods have been placed.

Tier Two methods may have a limitation(s) that make(s) them less accurate, precise, or sensitive, or less easily used than the Tier One methods, or they were simply not evaluated in depth in this article. That is not to say (nor to imply) that data generated with Tier Two methods will be less useful for industrial hygiene, epidemiological, enforcement, or liability studies.

The single most important factor in each evaluation is: Can the method serve the purpose of generating accurate and precise data at and below the ACGIH ${ }^{\circledR}$ TLV ${ }^{\circledR}$ for TDI vapor, or any other relevant occupational exposure limit (OEL)? The data must be obtainable on the basis of:

- a 4-8 hour time-weighted average (TWA),

- a 15-minute time-weighted average for the short-term exposure limit (STEL), and

- for shorter periods of time in the case where a production process may emit very short transients of TDI vapor that may or may not exceed the STEL.

Since the TLV TWA is $5 \mathrm{ppb}\left(36 \mu \mathrm{g} / \mathrm{m}^{3}\right)$, and the TLV STEL is $20 \mathrm{ppb}\left(140 \mu \mathrm{g} / \mathrm{m}^{3}\right)$ the method(s) must be capable of achieving these levels. Furthermore, some fraction of these levels must be achievable (both on a shorter time-basis and on a lower concentration-basis) under routine use.

\section{TIER ONE: PRIMARY METHODS EVALUATED IN THIS ARTICLE}

\section{OSHA Method 42 and Variants Thereof ${ }^{(15-21)}$}

This is the method that is most widely used and represents the current state-of-the-art against which all other methods must be measured. Because OSHA uses this method, there is a great incentive for all companies in the United States to use it. This method uses 1-(2-pyridyl) piperazine (PP) on a filter, with solvent desorption in the laboratory and HPLC analysis.

Samples are collected by drawing a known volume of air through glass fiber filters coated with $0.1 \mathrm{mg}$ of 1-(2-pyridyl) piperazine that are contained in open-face cassettes. Samples are extracted with 90/10 (v/v) acetonitrile/dimethyl sulfoxide 
(ACN/DMSO) and analyzed by high performance liquid chromatography (HPLC) using an ultraviolet (UV) or fluorescence detector. ${ }^{(15-18)} 1-2$ PP is a suitable derivatizing reagent, when coated on a glass fiber filter, for several reasons:

1. The high boiling liquid is retained on a glass fiber filter and stability is not a problem.

2. The rapid and exothermic reaction with both aromatic and aliphatic diisocyanates results in derivatization on the filter.

3. The derivatives have higher molar absorptivities in the UV region than those formed with nitro reagent, which allows the extraction volume to be larger without loss of sensitivity.

The combination of the PP reagent, the diisocyanate-PP derivatives, and the HPLC method allow for the baseline separation of all possible monomer analytes in a single run, without the need for fraction cutting and re-chromatographing. This means that HDI, HDI biuret trimer, TDI, MDI, HMDI, IPDI, and PAPI can all be identified and quantified in a single run (Figure 1). This is a significant strength of this method. ${ }^{(17)}$

Many laboratories use OSHA Methods 42 and 47. Anecdotal information indicates that minor variations in the method exist. Evaluation studies have been performed by many groups. ${ }^{(18-21)}$

\section{Considerations in the Use of this Method}

1. Amount of PP Reagent: A modification that may be used is to coat the filter used for sample collection with $2.0 \mathrm{mg}$ of PP reagent (as is the practice at Bayer ${ }^{(20)}$ ) or $10.0 \mathrm{mg}$ of PP reagent (as is the practice at Dow ${ }^{(21)}$ ), rather than the $0.1 \mathrm{mg}$ of PP required by the OSHA method. In actual practice, OSHA uses $1.0 \mathrm{mg}$ of PP on the filters, rather than the $0.1 \mathrm{mg}$ reported in the published method. Experiments show that, on hot and humid days, this additional
PP reagent is required in order to obtain accurate results if air volumes of up to 240 liters are taken during sampling. The use of filters coated with inadequate amounts of PP may represent a limitation when the $0.1 \mathrm{mg}$ called for in the original OSHA method is used under hot and humid conditions. Use of additional PP on filters and during desorption and analysis has no deleterious effects on the HPLC chromatogram.

2. Filter Backup Pad: The OSHA method calls for the use of a mixed cellulose ester (MCE) backup pad. The Bayer corporate method 1.7.6 variant of OSHA method 42 utilizes $2.0 \mathrm{mg}$ of PP on the filter and a stainless steel backup pad to avoid extraction of the PP from the coated filter to the backup pad. This method reportedly reduces the loss of PP from the filter during sampling from 75 percent in four hours to near zero percent loss when using the stainless steel pad. ${ }^{(20)}$

3. Filter Stability: It has been reported anecdotally that preprepared PP filter cassettes may lose some significant fraction of the PP to the walls of the cassette, thereby rendering the filter coating inadequate. The method used by OSHA is to

a) prepare the coated filters a minimum possible time prior to use;

b) store the filters in a bulk stack under refrigeration at $4^{\circ} \mathrm{C}$;

c) ship the filters as a stack of 10-25, or the amount needed if greater, in a minimal-size Nalgene or polystyrene bottle (no refrigeration during shipment); and

d) the end user stores the bottle of filters under refrigeration until use.

It has been reported that, if this procedure is used, the filters are undegraded for up to 2.5 years. ${ }^{(17)}$

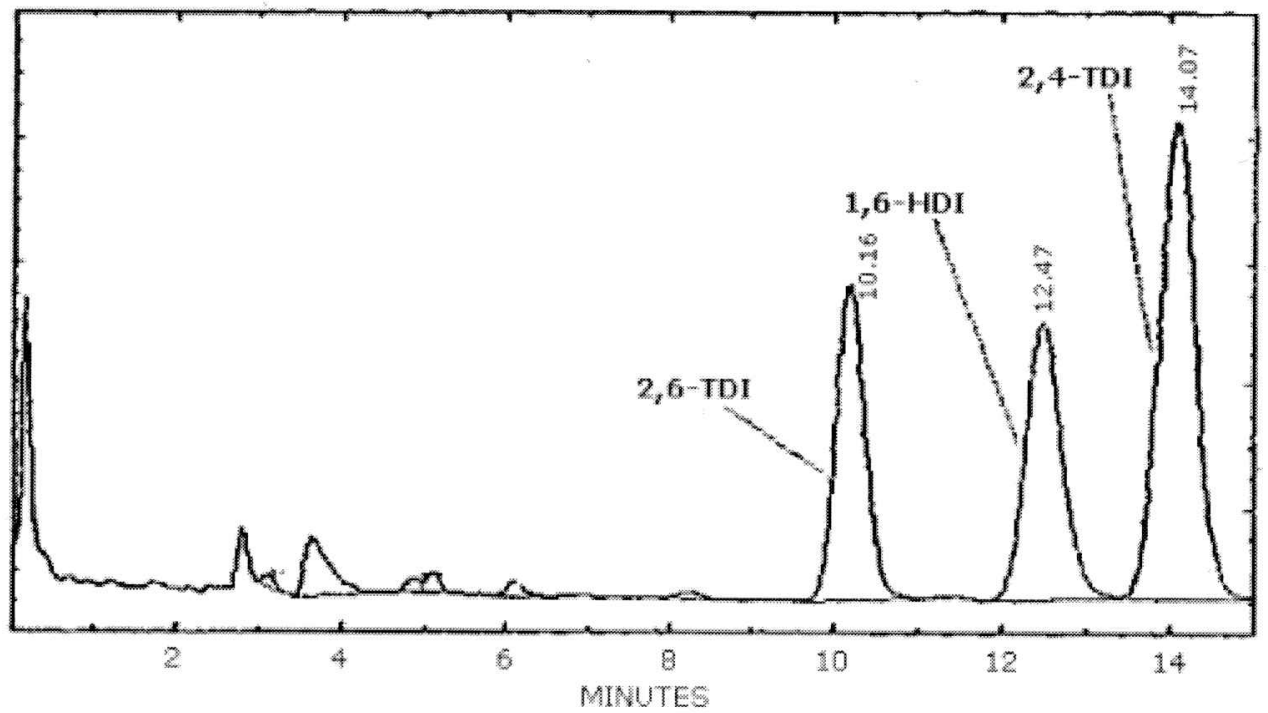

FIGURE 1

HPLC-UV chromatogram of toluene diisocyanate (TDI) and hexamethylene diisocyanate (HDI) using OSHA Method 42. 


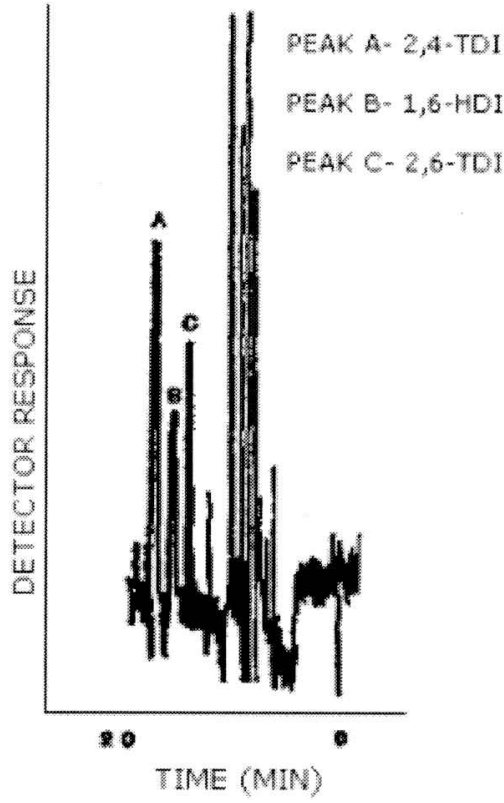

FIGURE 2

Portion of HPLC-UV chromatogram of TDI and HDI using OSHA Method 42. Same as Figure 1, except performed at the limit of detection of the method.

4. TDI Recovery: There have been no reports of degraded recovery for TDI vapors, when comparing extraction of the filters in the laboratory versus immediate field extraction. Anecdotal evidence indicates that there is no loss of TDI caused by waiting for laboratory extraction. ${ }^{(19,20)}$

5. Detector: All published data are for HPLC analysis using a UV detector. When using the UV detector, the baseline noise is quite high at the quoted LOD of the method (Figure 2).

However, the use of a fluorescence detector is advantageous because of the improved baseline noise at high sensitivity settings. Since PP is only weakly fluorescent, OSHA reports that the improvement in LOQ when changing from a UV absorbance to a fluorescence detector is only about a factor of two. ${ }^{(17)}$

The OSHA Salt Lake City laboratory uses a gratingfilter fluorescence detector. Excitation is set at $240 \mathrm{~nm}$. The emission wavelength is chosen with a $370 \mathrm{~nm}$ filter. OSHA has not performed a systematic evaluation of the improvement in the LOD or LOQ using this detector. NIOSH tests indicate that the gain in LOD/LOQ is approximately a factor of two better than the results obtained with the UV detector. ${ }^{(17)}$

Bayer, which uses a state-of-the art grating-grating instrument, reports a factor of five improvement in LOQ. As can be seen in Figure 3, the peaks at concentrations just above the LOQ are very large. The statistical basis for calling such a chromatogram that of a near-LOQlevel signal are extremely conservative, and incorporate measurements of variance from all aspects of the sampling and analysis. It is highly likely that other laboratories would quote a much lower LOQ if they were able to achieve a chromatogram of this signal/noise ratio. Chromatograms at the LOQ are just as noise-free as is shown in Figure 3 (which is a chromatogram of a quality control standard $).{ }^{(19,20)}$

6. Supporting Evaluation Data: The OSHA SLC laboratory has done a superb job of developing and documenting all relevant quality metrics for this method. Many other users have similarly documented relevant QC parameters.

Conclusions: Suitability of Method for TLV-Level Applications

When using a UV detector, this method is reported to have an LOQ of $0.4 \mu \mathrm{g} / \mathrm{sample}$, which translates to $0.2 \mathrm{ng} / \mathrm{HPLC}$ injection. Assuming a $2.0 \mathrm{~mL}$ sample solution, this means that the sample on the filter must contain $0.4 \mu \mathrm{g}$ that must have been collected from the air sample.

The original OSHA method called for a 15-minute sample totaling $15 \mathrm{~L}$ of air, which translates into an airborne LOQ concentration of TDI vapor of $3.8 \mathrm{ppb}\left(27 \mu \mathrm{g} / \mathrm{m}^{3}\right)$. Thus, this method, if used as originally validated by OSHA, and not counting the gains made through the use of increased PP loading, a stainless steel backup pad, and a fluorescence detector, meets the criterion of having an LOQ approximately one-fifth of the TLV STEL for a 15-minute sample.

This also means that a three-minute transient exposure of $20 \mathrm{ppb}\left(140 \mu \mathrm{g} / \mathrm{m}^{3}\right)$ or more will be detected and quantified by this method.

If the fluorescence detector is used, the method is capable of detecting approximately $1-2 \mathrm{ppb}\left(7-13 \mu \mathrm{g} / \mathrm{m}^{3}\right)$ in 15 minutes (depending on the detector), which is less than one-tenth of the TLV STEL.

This also means that a 3-minute transient exposure of $5-10 \mathrm{ppb}\left(70-140 \mu \mathrm{g} / \mathrm{m}^{3}\right)$ or more will be detected and quantified by this method, if a fluorescence detector is used.

If the improvements of $2.0 \mathrm{mg} \mathrm{PP}$ on the filter and a stainless steel backup pad are factored in, then up to $240 \mathrm{~L}$ of air can be taken. This means that the method can be used to sample continuously at $1.0 \mathrm{~L} /$ minute for 4 hours. Thus, this method satisfies all of the needs for both TLV TWA, TLV STEL, and, in essence, for all OEL-C criteria that are in force or have been proposed.

\section{NIOSH's MAP Draft Method 5525}

This method, developed by Robert Streicher and his group at NIOSH, is the newest of the methods..$^{(7,22,23)}$ Literature on its evaluation and use is just now being published. The original incentive for NIOSH to develop a new method was the TRIG problem. Although TRIGs are not considered in this article, this method is still worthy of review because of its direct and advantageous applicability to TDI vapor.

The NIOSH draft method 5525 differs from other methods in two important aspects: the reagent and the chromatography. The novel reagent 1-(9-anthracenylmethyl) piperazine (MAP) 


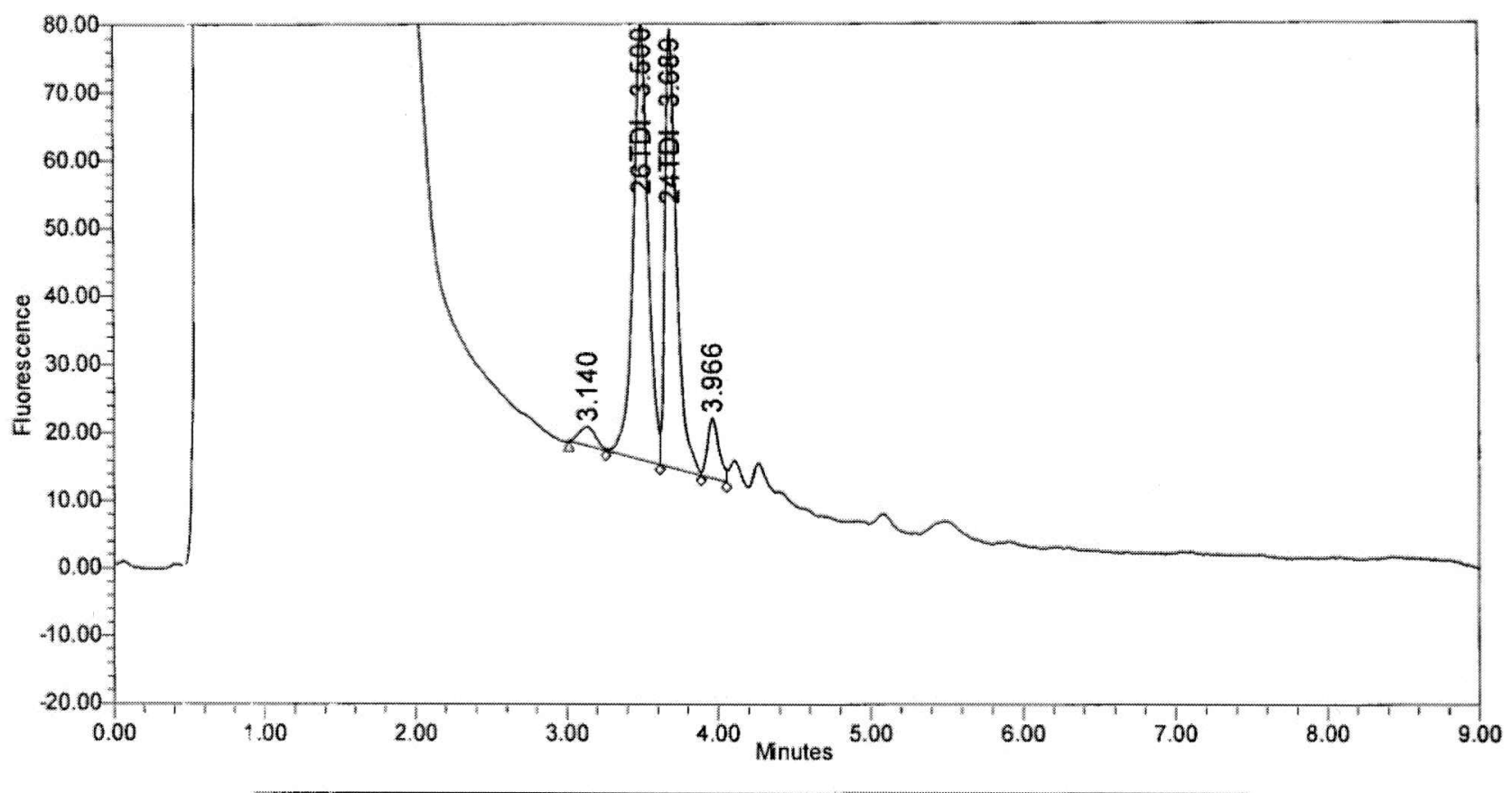

FIGURE 3

Quality control sample analyzed with fluorescence detection. Concentration is at three times stated limit of quantitation of $0.044 \mu \mathrm{g} / \mathrm{mL}$. Sample contains $0.12 \mu \mathrm{g} / \mathrm{mL}$ of $2,6 \mathrm{TDI}$ and $0.11 \mu \mathrm{g} / \mathrm{mL}$ of $2,4 \mathrm{TDI}$.

was shown to give better sensitivity and selectivity, greater uniformity in the UV response across different isocyanate species, and improved reactivity compared to the majority of commercial derivatizing reagents. Other advantages of MAP include substitution of the electrochemical detector with the more robust fluorescence detector and utilization of a $\mathrm{pH}$ gradient in the analysis.

The $\mathrm{pH}$ gradient for the isocyanate derivatives of MAP has several advantages compared to the commonly used organic modifier gradients. Because MAP derivatives contain a highly basic tertiary amine group, the retention of isocyanate derivatives of MAP is highly dependent on the $\mathrm{pH}$ of the mobile phase. This is especially important for high molecular weight prepolymers with multiple derivatized NCO groups, since the $\mathrm{pH}$ gradient will enable their elution from the analytical column within a very short time. For example, MDI is accelerated more than 100 times in a $\mathrm{pH}$ gradient. The $\mathrm{pH}$ gradient generates a more stable baseline, and column re-equilibration between runs is noticeably shorter. Furthermore, the $\mathrm{pH}$ gradient helps in minimizing interferences because the MAP-isocyanate derivatives respond to the $\mathrm{pH}$ changes of the mobile phase, but interfering compounds without the amine functionality do not. Overall, the strong $\mathrm{pH}$ gradient has the potential to produce much better chromatography, enabling a more accurate determination of total isocyanate species.

MAP contains the anthracene group, which is a strong chromophore and fluorophore. The anthracene group of MAP exhibits a primary UV absorbance band at around $256 \mathrm{~nm}$, and three weaker ones at around $352 \mathrm{~nm}, 368 \mathrm{~nm}$, and $388 \mathrm{~nm}$. The molar absorptivity coefficient of the $256 \mathrm{~nm}$ band is on average 18.1 times stronger than the $368 \mathrm{~nm}$ band, the strongest of the three weaker absorbance bands. MAP also possesses strong fluorescent properties, with emission bands at $396 \mathrm{~nm}, 418 \mathrm{~nm}$, and $442 \mathrm{~nm}$. Lastly, it may be used in impingers or coated on filters. The high reactivity of TDI vapor will make this an ideal candidate for sampling and analysis by this method (Table I).

However, only a very few papers have been published on the performance of MAP draft method 5525, and they focus mostly on the derivatization properties of MAP and its performance relative to that of other established reagents. Only now a very few laboratories are gaining experience with this method, and papers are being accepted for publication. Anecdotal evidence points to the potential for difficulty with certain complex aspects of the analysis method. ${ }^{(20)}$ Until further experience is gained, the decision to move to the MAP method from other methods should be done with caution.

The advantages of chromatographic flexibility and power, and detector response features, speak well for the idea that this method may be extensively used in the future. The quoted LOQ for this method is in the mid-ppt range $\left(\mathrm{ng} / \mathrm{m}^{3}\right)$, thus satisfying every conceivable need for sensitivity, short sampling times, and specificity.

\section{The ISO-CHEK Method}

In February 2001, an ISO-CHEK/OSHA 42 evaluation report was issued by Professor Roy Rando for the International Isocyanate Institute. ${ }^{(24)}$ Relevant literature references concerning 
TABLE I

Detector response factors and ratios of FLD, $\mathrm{UV}_{254}$, and $\mathrm{UV}_{370}$ for MAP ureas of some selected isocyanate species defined over a wide range of concentrations on standards and bulk products. Response factors of $\mathrm{UV}_{254}$ represent the slope of the calibration curve with at least four data points and $\mathrm{R}^{2}>0.99^{(7,22)}$

\begin{tabular}{|c|c|c|c|c|c|c|c|}
\hline \multirow[b]{2}{*}{ Isocyanate moiety } & \multirow{2}{*}{$\begin{array}{c}\mathrm{UV}_{254} \begin{array}{l}\text { response factor } \times 10^{-7} \\
\left(\mathrm{~S} . \mathrm{E} . \times 10^{-5}\right)^{\mathrm{A}}\end{array}\end{array}$} & \multicolumn{3}{|c|}{$\mathrm{FLD} / \mathrm{UV}_{254}$} & \multicolumn{3}{|c|}{$\mathrm{UV}_{254} / \mathrm{UV}_{370}$} \\
\hline & & Mean & $\mathrm{SD}^{\mathrm{B}}$ & $\mathrm{N}$ & Mean & $\mathrm{SD}^{\mathrm{B}}$ & $\mathrm{N}$ \\
\hline HDI monomer & $9.43(3.96)$ & 26.6 & 1.7 & 22 & 17.3 & 1.4 & 22 \\
\hline IPDI-1 monomer & $9.14(1.69)^{\mathrm{C}}$ & 31.9 & 0.9 & 11 & 17.1 & 0.3 & 12 \\
\hline IPDI-2 monomer & & 28.0 & 1.6 & 11 & 16.5 & 1.1 & 9 \\
\hline 2,6-TDI monomer & $9.41(7.68)$ & 49.1 & 1.0 & 5 & 16.6 & 0.9 & 7 \\
\hline 2,4-TDI monomer & $9.16(6.15)$ & 46.9 & 1.3 & 4 & 17.6 & 0.6 & 7 \\
\hline MDT (trimer of MDI) & $9.83(9.01)$ & 18.3 & 0.88 & 4 & 18.9 & 1.0 & 4 \\
\hline 8 different aliphatic bulk products & - & 21.3 & 7.4 & 145 & 16.1 & 1.1 & 38 \\
\hline Mean $(\mathrm{CV} \%)$ & $9.39(3.0)$ & $31.7(37.8)$ & & & $17.1(5.4)$ & & \\
\hline
\end{tabular}

${ }^{\text {A }}$ Standard error of the regression line coefficient.

${ }^{\mathrm{B}}$ Standard deviation.

${ }^{\mathrm{C}}$ For IPDI-1 and 2.

the ISO-CHEK method are cited in Rando's report. Evaluations were compared to results obtained using the Tulane dichotomous sampler, which served as a benchmark, and using a Zellweger/ MDA 7100 continuous monitor. Although this report dealt with TDI, MDI, and prepolymers, the results obtained with TDI vapors are the only ones that will be discussed here.

The ISO-CHEK method utilizes a $37-\mathrm{mm}$ cassette with a 5-micron Teflon pre-filter and a methylamino-methylanthracene (MAMA)-treated glass fiber filter. The design is aimed at achieving quantitative trapping of nonvolatile isocyanate species on the Teflon pre-filter, while vapor-phase isocyanate monomers pass through and are trapped and derivatized on the MAMA filter. Immediately after sampling, the Teflon filter is removed from the cassette and treated with a toluene solution of methoxy-phenylpiperazine (MOPP). The MAMA-derivatized samples are analyzed using UV/fluorescence detection. The MOPP samples are analyzed using multiwavelength UV detection.

For each test atmosphere, six samples were collected under identical conditions. For TLV TWA evaluations, sample atmospheres were generated at multiples of the $5 \mathrm{ppb}^{\mathrm{TLV}}{ }^{\circledR}$ for TDI vapor, for three-hour periods. For TLV STEL evaluations, sample atmospheres were generated at multiples of the $20 \mathrm{ppb}$ STEL for TDI vapor. The ISO-CHEK was reported to have a negative bias of 45 percent. The decrement is greater the longer sampling periods and is worse for TDI than for MDI. This method also inaccurately apportions collected isocyanate monomer into vapor and aerosol phases.

Whatever the reason(s) for these results and the disagreements that may be raised, this simple fact makes it all moot: For TDI vapor, the method is unnecessarily complex. In light of the strengths of modified OSHA Method 42 and NIOSH draft method 5525, it is unclear why the ISO-CHEK would be used for sampling TDI vapor.

\section{The 1-(2-Methoxyphenyl) Piperazine Method}

This method is designated as U.S. NIOSH Method 5521 and U.K. Method MDHS 25/3. It uses 1-(2-methoxyphenyl) piperazine (MOPP, also abbreviated MP) with HPLC analysis of the derivatized analyte. ${ }^{(25-28)}$ It is applicable both using impingers and MP-impregnated glass fiber filters. This method has seen, and is seeing, widespread use. MDHS 25/3 method is appropriate for a wide range of organic compound s containing isocyanate functional groups such as TDI, MDI, HDI, and IPDI.

Since quantitation is by direct analysis of the derivative rather than by calculation of the difference between the two numbers, this method eliminated some of the limitations seen with Method 5505. The method should perform in a quantitative fashion for individual isocyanates. As can be expected, all previously noted limitations of impingers exist for this method.

In MDHS 25/3, a measured volume of air is drawn through a glass impinger containing 1-2 MP solution and/or a glass fiber filter impregnated with the 1-2 MP reagent. Any organic isocyanates present will react to form a nonvolatile urea derivative. The resultant solution is concentrated and analyzed by HPLC with UV and EC detectors in series.

Isocyanate-derived peaks are identified on the basis of their EC and UV responses and also by comparison with derivatized bulk. Quantification is achieved by comparison with the relative isocyanate monomer standard. The total isocyanate-in-air concentration is calculated from the sum of all the isocyanatederived peaks.

In the United States, a modified version of these methods is used by Huntsman Company. ${ }^{(28)}$ The Huntsman method uses either a filter or impinger to collect isocyanates. Diethylhexylphthalate (DEP) is also added to the filter to aid in solvation of the aerosols and vapors collected. Filters are field desorbed using $1 \mathrm{~mL}$ of $1 \mathrm{~mm} \mathrm{1-2} \mathrm{MP} \mathrm{in} \mathrm{dry} \mathrm{toluene} \mathrm{to} \mathrm{minimize} \mathrm{any}$ 
isocyanate losses and to fully solvate any free isocyanate and reacted isocyanate-MP derivative. Upon receipt in the lab, the isocyanate-MP derivative is dissolved in acetonitrile/water solution, chromatographed and detected with the fluorescence detector the primary detector, followed by the UV detector in series. The electrochemical detector, used by some laboratories, is generally thought to be less suitable than the fluorescence detector because of baseline and instability issues and electrode corrosion.

The filters are coated with $2 \mathrm{mg}$ of MP derivatizing agent to ensure complete capture and derivatization even during hot and humid days. The use of excess MP reagent does not interfere with the HPLC analysis. In terms of filter stability, all filter samples are field desorbed after sampling. The field desorbed versus lab desorbed study showed that lab desorbed filters underestimate MDI compared to field desorbed. This finding may also be extended to TDI, although data are not available supporting the need for field desorption of collected TDI vapor samples.

Gradient elution is used to separate monomer peaks from oligomer peaks instead of isocratic separation. It also removes all the compounds from the column. Of course, this will not be necessary for samples containing only TDI monomer.

The sensitivity, accuracy, and precision of the method are generally quoted as being equivalent to the modified OSHA Method 42. Why, then, are both the PP and the MP methods in use? It is because the PP method is OSHA's method of choice, and the MP method is HSE's method of choice. The PP method is also an official method of the International Organization for Standardization (ISO). ${ }^{(29)}$

\section{TIER TWO METHODS}

These are methods that will be reviewed in this article only briefly.

\section{Marcali Method}

The first method encountered in actual use, both historically and until the near-present is the Marcali method. This method uses an impinger with a solution of hydrochloric and acetic acids for sample collection, and N-1-naphthylethylenediamine for colorimetric analysis of the impinger solution in the laboratory. It is designated as NIOSH Method $141 .^{(30-32)}$ The method has the following characteristics (all statements are taken from references 30-32 unless indicated otherwise).

There is a positive interference from aromatic amines. This may be important in any case where aromatic amines may be present in the air. There is no specificity for individual isocyanates. This is not a problem when only a single isocyanate is present. 2,4- and 2,6-TDI have different absorption coefficients, with the coefficient for 2,4- being higher than 2,6-TDI at the wavelength used for this method. Studies in foam plants have shown that 2,4- predominates in the initial stages of the process, and 2,6- predominates during the end stages. TDI commonly used in processes may be 80 percent $/ 20$ percent or 65 percent/ 35 percent 2,4-TDI/2,6-TDI isomer mixtures. In some cases, standards used for the analysis are 100 percent 2,4-TDI. In 1985, Rando et al. reported a modified Marcali method to deal with this issue. Therefore, depending upon the circumstances, results for TDI exposure that did not use this modified method may be subject to some error and need to be interpreted with caution.

There may be insufficient sensitivity for taking short-term samples at low concentrations. This question of sensitivity (really of limit of detection [LOD] and quantitation [LOQ]) is important when a link is sought between a specific process state or worker action and exposure. Because of the relative insensitivity of the Marcali method, it may not be possible to establish these links.

The quantitative range of the method is about $7-140 \mathrm{ppb}$ for TDI based on a 20-liter air sample. The LOD of this method can be reduced further by minor methodological changes. It is important that any study using this method be checked to ensure that quantitative results have not been given below these values unless validation has been performed. However, a precision of $+/-5$ percent can be achieved at $20 \mathrm{ppb}$. It has been demonstrated that impinger efficiency for TDI vapor is $90-95$ percent when using the Marcali method.

\section{Nitro Reagent Method ${ }^{(33-35)}$}

This is designated as NIOSH Method 2535, OSHA Method 18, and Bayer Method 1.4.4. In the NIOSH method, a sampling tube is used with p-nitrobenzyl-N-n-propylamin e (nitro reagent) deposited on glass wool and packed in a sampling tube. While the nitro reagent methods have been widely used for sampling aliphatic isocyanates, this method has seen little use in recent years for aromatic isocyanates. There is no advantage in using this method for TDI.

The nitro reagent is also only good for a maximum of 10 days, subsequent to preparation, as long as it is kept in the dark. Tubes should be wrapped with black tape to keep out light. Decomposition of the reagent results in spurious peaks that interfere with the HPLC analysis.

In the OSHA method, p-nitrobenzyl-N-n-propylamin e (nitro reagent) in an impinger with HPLC analysis of the derivatized analyte is used. The high flow rate of sampled air through the impinger results in significant loss of toluene solution. This toluene must be replaced during sampling.

In addition, if the toluene reagent is not completely evaporated in the laboratory prior to analysis, the toluene gives a positive HPLC interference with the 2,6-TDI peak. The method LOQ is $2.4 \mu \mathrm{g} / \mathrm{m}^{3}$ for TDI ( $0.3 \mathrm{ppb}$ each) when 20 liters of air is sampled. The LOD/LOQ must be adjusted for the volume of air actually sampled.

\section{NIOSH Method 5505}

This method uses 1-(2-methoxyphenyl) piperazine (MOPP) in an impinger. After the sample is collected, and the isocyanate has been derivatized with the MOPP, HPLC analysis of the remaining MOPP reagent is performed. ${ }^{(36)}$

Since quantitation is accomplished by comparing the difference between the original amount of MOPP and the final lesser 
amount, there is a significant degree of uncertainty in measuring a small difference between two large quantities. The uncertainty is magnified when consideration is given to the possible loss of MOPP reagent by solvent entrainment in the air flow through the impinger. Also, there is an unacceptably high bias in the results due to MOPP reagent loss.

Health effects studies using this method should not be considered to have quantitative exposure results. This method was formally retracted by NIOSH. ${ }^{(37)}$

\section{The HPLC-Mass Spectrometric (MS) Method ${ }^{(38)}$}

Skarping et al. have published a number of papers, many of which have been cited in reference 38 . This paper purports to demonstrate two things: 1 ) that the MOPP method (reviewed above) results in data that have a significant low bias when compared to di-n-butylamine (DBA) derivatization, and that this is due to the higher reaction rate of DBA with isocyanate functional groups; and 2) the HPLC-MS method is a viable alternative for routine analyses when compared to HPLC used with other detectors. Neither of these hypotheses is convincingly demonstrated in this paper.

First, investigations of bias, or other quality metrics, and/or reaction rate constants must be accomplished in strictly controlled laboratory settings in which every relevant parameter has been systematically varied over a known range. In the referenced paper, Skarping did not do this.

Second, it is profoundly counterintuitive to believe that HPLC-thermospray MS is a method as simple, reliable, and generalizable as is HPLC-UV or HPLC-fluorescence. The complexity and cost of the instrumentation argue convincingly against this approach unless a clear advantage is to be gained in sensitivity, specificity, or some other parameter.

That is not to say that the HPLC-MS might not be very useful for certain applications involving speciation of complex analytes. This is not the case for analysis of TDI.

\section{AIR MONITORING INSTRUMENTS ${ }^{(1-8,39-47)}$}

There is a very clear case of a "good news-bad news" dichotomy for real-time monitoring instruments for TDI vapor. The good news is

- Several instruments are available for fixed-point alarm or hand carried survey instrument purposes.

- The instruments are, in general, capable of providing short sampling time, high sensitivity results either for survey or alarm.

- The critical design features of these instruments have evolved sufficiently to have solved some critical issues that might lead to inaccurate results.

- Evaluation data are available from some users of some of these instruments.

- Many of the problems discussed in the literature are limited to the application of these instruments to MDI and to diisocyanate-containing aerosols, but these problems are not applicable to the monitoring of TDI vapor.

The bad news is

- There is a paucity of readily available, current, peerreviewed published evaluation data, from university, government, or manufacturers that is especially glaring given the importance attached to their use.

- Some of the best evaluation data are available for instruments that are no longer used.

- For some applications, the accuracy of certain instruments may be both poor and variable at lower concentrations.

- There is no longer a continuou s personal exposure monitor commercially available.

- Non-paper tape, alternate technology has been slow to evolve.

Of all of these positive and negative points, the single most critical conclusion is that these are extremely important tools in our arsenal of approaches to industrial hygiene exposure assessment and alarm for diisocyanate vapors. The use of these instruments must be encouraged, despite the above shortcomings.

Interestingly, there was a flurry of activity on this important subject area in the mid-1980s that led to significant publications by Rando, Dharmarajan, and others, much of which was published in peer-reviewed journals. The fact that this work has not been repeated in recent years, as a high priority project funded by NIOSH, is unfortunate. However, some continuing work is ongoing in the laboratory of Dharmarajan and others, funded by corporate or International Isocyanate Institute budgets. With the lack of resources at NIOSH, and the steady shrinkage of manpower at industrial research labs, it is unlikely that additional work will be done in a timely, comprehensive fashion.

Some of the most comprehensive evaluation work has been performed on the Zellweger/MDA Isologger, which unfortunately is no longer made. ${ }^{(48,49)}$ This instrument presumably uses the same paper tape chemistry and the same detector system as other Zellweger/MDA instruments that are still produced, so the study has intrinsic value that goes beyond the specific instrument. Zellweger/MDA has stated that the suspension of production of this instrument, and of the related miniature continuous monitor (MCM) had nothing to do with the design or operating characteristics of the instrument. Rather, discontinued instruments are no longer produced because obsolete parts are no longer available, and the market drivers for instrument redesign were not always there. That is, the demand did not justify the re-engineering cost.

Dharmarajan found that, at high humidity $(80 \%)$, the Zellweger/MDA instrument had a negative bias of about 40 percent at concentrations of $19 \mathrm{ppb}$ and above. At concentrations of $15 \mathrm{ppb}$ and below, the instrument had a positive bias of about 40 percent. At low concentration (5 ppb) and low humidity (50\%) the instrument had a positive bias of 17 percent. This is virtually 
identical to the results obtained in the study in Japan by Nakaaki, who reported that, at 68 percent humidity and $25^{\circ} \mathrm{C}$, the instrument gave higher values in the low concentration range and lower values in the high concentration range. ${ }^{(49)}$ Dharmarajan also studied a GMD instrument and found that it too had biases caused by changes in humidity, but that those biases were different in nature and direction from those of the Zellweger/MDA instrument.

Studies of the tape chemistry and the effect of $\mathrm{NO}_{\mathrm{x}}$ and ozone on response have been performed by D' Arcy of General Motors for HDI, but not, to our knowledge, for TDI. It appears that the Zellweger/MDA tape chemistry's response is strongly affected by the presence of oxidizers. ${ }^{(20,50,51)}$ The tape chemistry used by the GMD/Scott/Bacharach instrument is also affected by oxidizers as well as by extremes of humidity, but possibly significantly less so than the Zellweger/MDA tape chemistry, although peerreviewed data to support this contention is not available for the many tape chemistries.

The best review of this literature will be available in late 2002 in a book by Dharmarajan entitled Safe Handling of TDI and $M D I{ }^{(8)}$ Dharmarajan has stated that, “... when questions of [oxidizer] interferences arise, the industrial hygiene chemist on site should physically examine the previously exposed tape. If the tape is the characteristic brick red color, the response is probably due to TDI ...." ${ }^{(8,20)}$ (An orange-hued color is typical of $\mathrm{NO}_{\mathrm{x}}$ interference.) This is an important point in that it highlights the need for trained industrial hygiene chemists to prevent false positive results with the resultant misuse of inaccurate or questionable data that can undermine the confidence of companies and of the public. ${ }^{(12,50)}$

Other interferents, such as dust and diisocyanate-containing aerosols, are beyond the scope of this article, but are clearly subjects of concern. ${ }^{(8)}$

The GMD instruments also appear to have the potential of being more accurate throughout the concentration range. This appears to be supported by data from the studies by Dharmarajan, by Coyne et al. at Dow, and by Groves et al. at the U.K. HSE in 1985. The problem is that only one of Dharmarajan's studies is published, but the rest are only in internal company reports. The D' Arcy study (for HDI only) is available only from the author in PowerPoint form, the Coyne articles were never peer reviewed, but instead are reprints of her slides from a talk, and the HSE data are contained in reports rather than in widely disseminated peerreviewed articles, so relatively few people see this information.

To what can we attribute the possibility-unproven as it isof superior performance of the GMD instruments? There are two possible factors that seem obvious: The first is that the tape chemistry may indeed be different enough in the instruments of the two companies to account for the big difference in response. Such information is not readily available, so sound conclusions cannot be drawn on this subject.

The second is that the Zellweger/MDA instrument design may be older. The more recent design of the GMD instrument employs a variable sampling time method in which the rate of change of the colored spot on the tape is read, and the tape is stepped forward more quickly if the rate of change of the spot density with time is higher than a certain set point. This tends to keep the loading of TDI vapor on an individual spot in the optimal range for the most linear, or most accurate, portion of the tape color response. At first glance, it might be logical to believe that there is the possibility that the GMD instrument design might result in more accurate results, all other things being equal.

However, data available from Zellweger/MDA show that their instruments - the Isologger, 7100, TLD, and SPM-have been well-characterized by the company, and in their laboratory-based tests are capable of high-speed, high-accuracy, low-LOQ results that are more than adequate for most monitoring needs. ${ }^{(51)}$ The question of the GMD "rate of change of spot density" method versus the Zellweger/MDA "absolute value of spot density" method is poorly characterized and clarification is impeded by confidential, proprietary design features of the instruments, with the potential for variation in design being both between and within instruments and companies.

The single most serious issue that the data show is that there must be a substantial correction factor for humidity. The Zellweger/MDA instruments have both normal and high humidity response curves that can be user-selected. It is not clear that this user-selected, two-curve system really works, because the selection criteria may break down in the vicinity of the 7085 percent relative humidity range. This may account for the difference in the data published by Dharmarajan's team and the data supplied by Yamaguchi. $(20,48,51)$

Given the availability of inexpensive computing power, it is unclear why all such instruments do not incorporate both an electronic hygrometer and a family of response curves that are automatically employed by the instrument. This would solve a host of problems. In fact, an electronic "total oxidizer" detector could also be incorporated in such a design. To critics who say "That would add cost," the response must be that inaccurate results are inherently expensive, and microminiaturization has led to the commodization of such detectors to the point where the added cost, after the design cost is amortized, would be minimal. However, a significant cost would be (as is frequently the case) associated with the evaluation of instrument performance parameters, both in the laboratory and in the field.

Additional computing power would also help to solve the asyet unevaluated issue of "what is the real LOQ under actual field conditions?" Too often, the untrained user of the instrument will take a continuum of digitally displayed results in the low-ppb concentration range and combine those "quantitative results" with a decision-making process based on ever-lowering OELs for workers or the community. This may lead to distorted results and incorrect decision making. ${ }^{(12)}$

The paper tape instruments currently marketed have the drawback of being fixed systems, semi-portable, or at best handportable. When one considers the importance of measuring concentrations in the workers' breathing zone, these instruments 
should be used cautiously as indicators of personnel exposure. However, they do have the advantage of recording concentration over time, down to 30 -second intervals. The importance of this is that the hygienist can have results immediately at the end of the sampling period. This allows him or her to query the worker for the activity that caused a "peak" of elevated exposure. Since industrial hygienists are concerned with recognition, evaluation, and control, these instruments are the ideal tools for measuring TDI and ultimately achieving control.

An additional category of devices is the passively or actively pumped sampling badge. After a pre-set period of time, sometimes less than 15 minutes, the badge is withdrawn from the air being sampled and visually measured against a color calibrator equipped with a color comparator for a range of given concentrations of TDI. The advantage of this is that the visual matching of colors and intensities gives the card reading an additional dimension - that of color measured by the human eye, rather than just "darkness" measured by a densitometer. The disadvantage of this relates to the intra- and inter-individual variability associated with a human measurement. Nonetheless, this method appears to work relatively well. This was demonstrated by the study of the GMD Sure-Spot pumped air sampling instrument by Milsom and Groves at the HSE in 1991. ${ }^{(44)}$

This same approach has been extended to passive sampling badges, and may work just as well. This is an excellent way to protect large numbers of workers at low cost. Of course, as with all passive methods, the limits of detection and quantitation will be higher than with pumped methods.

TDI dosimeter badges can be a valuable tool for the industrial hygienist. Worker acceptance is high, and badge programs, if set up properly, can practically run themselves, overseen by an industrial hygienist. They are most effective as a screening tool, using other methods to quantify exposure if there is indication of concern. An immediate answer is available based on a visually evident characteristic color change. The LOD for the K\&M Safeair passive dosimeter system is 2.5 ppb-hours for a 15-minute (or less) sampling period, and 5 ppb-hours for longer time periods. The relationship between the LOD and the LOQ is uncertain for this device, as it is for all visually read devices.

The reason that this badge should be used primarily as a screening tool is that it is not a true "Fick's First Law" device. There is no design geometry that ensures only diffusion-limited pumping. However, at air flow rates of about $40 \mathrm{~cm} / \mathrm{second}$ and below, there is a quantitative relationship between badge exposure and measured color density. At low flow rates, there is little evidence of a starvation effect frequently seen with true Fick's Law devices. At higher flow rates, there is an increasingly positive bias to the results. ${ }^{(52,53)}$

What is the practical result of this? First, in indoor or low air flow rate environments, the results obtained with this device will be quantitative and can be used for quantitative exposure assessment. At higher flow rates, the results will have a high bias, which may result in overprotection of the workers, or, in epidemiological studies, in overestimation of exposure. There- fore, as with most such devices, an industrial hygienist must set up the monitoring program and interpret the data.

Lastly, the K\&M passive monitor uses a chromophore coated on a plastic film. Apparently, this results in improved resistance to bias from oxidizers and improved storage stability when compared to paper tapes. However, there is no comparative peerreviewed data available on this subject.

A caveat to all of these analyses is that tape chemistries and instrument design features are always evolving, and instrument manufacturers may or may not have data that indicate improved performance in newer instruments. Without a prioritized effort to perform peer-reviewed quality evaluation of these newer instruments, the industrial hygienist will not have the ability to optimize the accuracy of results.

There is little indication, other than at the International Isocyanate Institute, that these issues are getting the priority they deserve. For example, the laboratory that developed OSHA Method 42/47 did so in the 1980s when their manpower level was at 8 full-time equivalent (FTE) employees. Now they are at 3 FTEs. The laboratory that developed the NIOSH methods did so in the 1990s when their manpower level was at 35 FTEs. Now, they have 25 FTEs. In 5 years, they expect to have 14 FTEs. When these methods were first developed, the questions were simple and were of the type involving the airborne concentrations of a few monomeric isocyanates. Now, the easy questions have been answered; those remaining are exceedingly complex.

Federal and state regulators regularly drive down PELs; $\mathrm{ACGIH}^{\circledR}$ drives down voluntary TLVs ${ }^{\circledR}$; the public demands OELs that are below odor thresholds, are protective all the time for all the population, and are applicable to all processes and products. Without such laboratory and field industrial hygiene person-power, improved real-time personal sampling instruments may not be developed, evaluated, or utilized properly.

However, it is clear that the use of these instruments has and will continue to serve a useful purpose, both for alarms and for exposure assessments. Caution must be used in interpreting quantitative results obtained with these instruments, unless specific validation data are available.

\section{QUALITY ASSURANCE AND CONTROL}

In addition to timely evaluation/validation studies, the industrial hygiene chemist must ensure that all QA/QC protocols are followed for the calibration of instruments and the treatment of data thereby obtained. Too often a digitized instrument output of many significant figures is transcribed and treated as gospel, without the proper critical evaluation of all relevant factors.

There are those who say "But how do we know that the reported results are accurate?" The answer is that there is a QA/QC system for isocyanate analysis. Although this system is based on MDI proficiency samples, a laboratory that performs a significant number of MDI samples will, in all likelihood, also perform many TDI analyses. Since the same factors affect 
accuracy, this QA/QC system will be almost as applicable to both compounds.

This system is based on PAT samples obtained through the British Workplace Analysis Scheme for Proficiency (WASP). Standard Shewhart Control Chart methods are used for statistical analysis of the results. When this is combined with the American Industrial Hygiene Association (AIHA) Laboratory Accreditation program, there is a high degree of assurance, and a recorded statistical basis of the results for TDI and MDI sampling and analysis. This is an objective basis upon which to base a presumption that such data are accurate because they come from a WASP-certified and/or AIHA-accredited laboratory. ${ }^{(54,55)}$

Such a laboratory upon which other labs can benchmark their results is the Huntsman Company laboratory run by Premal Parekh. In the latest WASP summary of results, they had no outliers and were ranked number one of 15 such labs in the world.

Other laboratories generate their own PAT samples and keep their own internal data sets for each method. Figure 4 shows two Shewhart quality control charts for TDI. Note that for any QC result outside the two sigma warning limit, the results from that
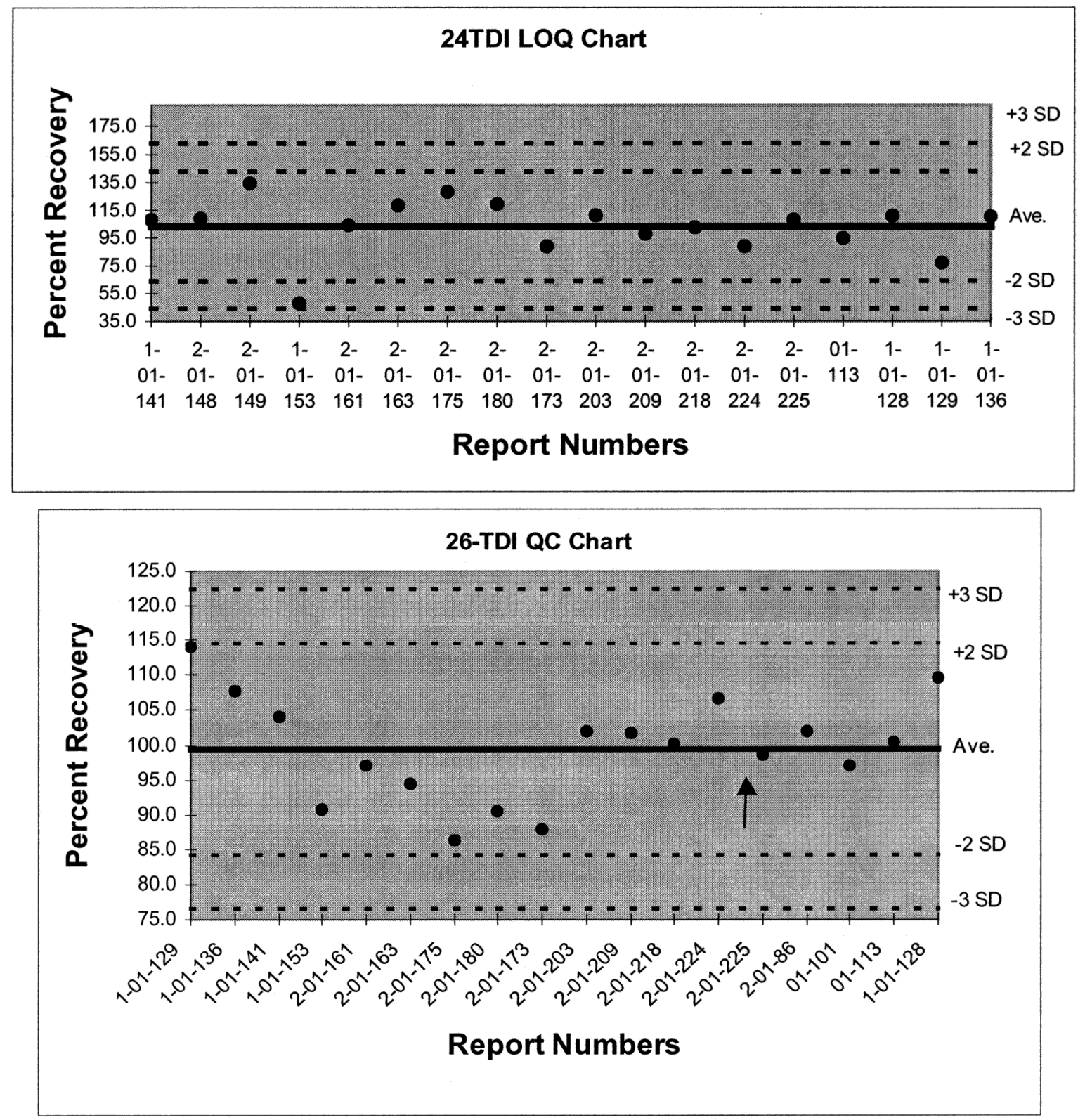

FIGURE 4

Shewhart quality control charts for overall recovery including all steps in sampling and analysis procedure. $+/-2$ SD indicates "warning limits." +/-3 SD indicates "control limits." Arrow in lower chart indicates point at which a periodic QC report is generated for continuous quality improvement. ${ }^{(20)}$ 
batch of analyses would be accepted, with an appropriate footnote to the data, an investigation into the cause(s) of the out of two sigma condition would be launched, and corrective action taken. For any QC sample with results outside the three sigma control limit (there are none in these examples), results of the analyses of the corresponding sample batch would not be acceptable. The arrow in the lower of the two Shewhart charts is the point at which a periodic quality control report was written. In this example, for every 18 analytical batches, such a report is written. Based on the results during this period and the conclusions in the report, corrective and preventive actions and continuous quality improvements are made. This is an exemplary program that should be benchmarked by other laboratories. ${ }^{(20)}$

\section{CONCLUSIONS}

Certain methods are more than capable of achieving results for LOQ far in excess of what is needed for any occupational exposure limit or sampling period. There are new methods, such as NIOSH draft method 5525, which may provide even better sensitivity and specificity, but must be more widely tested before being generally adopted as a benchmark.

Monitoring instruments, operated within validated parameters, are capable of sensitivity, speed, and continuous operation that make them invaluable. Improvements in instrument design could be implemented that would increase accuracy under a variety of field conditions.

Quality control and quality assurance systems are available for use, and should be used, for laboratories engaged in isocyanate analysis. In extreme cases, lack of proper quality control might result in the poor sampling, analysis, and data evaluation problems of the type recently reported by Levine et al. ${ }^{(12)}$ This must not be allowed to occur.

Now that the tools are available and well understood, they must be applied to well designed exposure assessment/ epidemiological studies. Occupational exposure limits should not be based on studies in which less-than-optimal exposure assessment methodology might have been used.

\section{ACKNOWLEDGMENTS}

The author thanks Robert Streicher of NIOSH, Donald Burright of OSHA, members of the American Chemistry Council's Diisocyanate Panel, and representatives of GMD and Zellweger/MDA instrument companies for their information, advice, and/or support.

The American Chemistry Council, Diisocyanate Panel, Washington, DC, supported this work.

\section{REFERENCES}

1. Purnell, C.; Walker, R.: Methods for the Determination of Atmospheric Organic Isocyanates: A Review. The Analyst 110:893-905 (1985).

2. Analysis of Isocyanates in Air: Technical Information Report \#5. International Isocyanate Institute, New Canaan, CT (1982).
3. Langhorst, M.; Coyne, L.: Industrial Hygiene (Application Reviews). Anal Chem 61:128R-142R (1989).

4. Dharmarajan, V.; Lingg, R.; Booth, K.; et al.: Recent Developments in the Sampling and Analysis of Isocyanates in Air. In: Calibration for Atmospheric Measurements. ASTM STP 957, J.K. Taylor, ed., pp. 190-202. American Society for Testing and Materials, Philadelphia (1987).

5. Levine, S.P.; Hillig, K.J.D.; Dharmarajan, V.: Critical Review of Methods of Sampling, Analysis and Monitoring for TDI and MDI. Am Indus Hyg Assoc J 56:581-589 (1995).

6. Streicher, R.P.; Reh, C.M.; Key-Schwartz, R.: Determination of Airborne Isocyanate Exposure. NIOSH Manual of Analytical Methods, pp. 115-140. NIOSH, Cincinnati, OH (January 15, 1998).

7. Streicher, R.P.; Reh, C.M.; Key-Schwartz, R.; et al.: Determination of Airborne Isocyanate Exposure: Considerations in Method Selection. Am Indus Hyg Assoc J 61:544-556 (2000).

8. Dharmarajan, R.; Madison, P.; and Brenner, K.: Safe Handling of TDI and MDI. Manuscript in preparation for publication, Dharmarajan, R., Bayer Corporation, Pittsburgh, PA, to S. Levine, personal communication (January, 2002).

9. Musk, A.W.; Peters, J.M.; Wegman, D.H.: Isocyanates and Respiratory Disease: Current Status. Am J Indus Med 13:331-349 (1988).

10. Weill, H.; Butcher, B.; Dharmarajan, V.; et al.: Respiratory and Immunologic Evaluation of Isocyanate Exposure in a New Manufacturing Plant. NIOSH Technical Report. Contract 210-75-0006, NIOSH, Morgantown, WV (1981).

11. Garabrant, D.H.; Levine, S.P.: A Critical Review of the Methods of Exposure Assessment and the Pulmonary Effects of TDI and MDI in Epidemiological Studies. Chemical Manufacturer's Association, Washington, DC. Also available as International Isocyanate Institute Report No. 11379, from: British Library Document Supply Centre, Boston Spa, Wetherby, West York, UK (1994).

12. Levine, S.P.; Redinger, C.F.; Robert, W.P.: Community Exposure Assessment and Intervention Effectiveness at Trinity of America Corporation, Glenola, NC. Am Indus Hyg Assoc J 62:649-657 (2001).

13. Daftarian, H.S.; Roegner, K.C.; Reh, C.M.: Woodbridge Corporation, Brodhead, Wisconsin, NIOSH Health Hazard Evaluation Report, HETA 98-0011-2801. NIOSH, Cincinnati, OH (2000).

14. Levine, S.P.: Metrological Recommendations for Air-Monitoring Instruments-A Guidance Document. AIHA Gas and Vapor Detection Systems Committee. Am Indus Hyg Assoc J 53:673-676 (1992).

15. OSHA Method 42: Diisocyanates. OSHA Analytical Laboratory, Organic Methods Development Branch, Salt Lake City, UT (1983).

16. OSHA Method 42: Diisocyanates. OSHA Analytical Laboratory, Organic Methods Development Branch, Salt Lake City, UT (March, 1989).

17. Burright, D.: OSHA Analytical Laboratory, Organic Methods Development Branch, Salt Lake City, UT, to S. Levine, personal communication (January 24, 2002).

18. Test Method D5836-95 Standard Test Method for Determination of TDI in Workplace Atmospheres (1-2 PP Method), ASTM Subcommittee D22.04. American Society for Testing and Materials, West Conshohocken, PA (2001).

19. Bayer CIHL Method No: 1.7.6. Revision 6. Bayer Corporation, Pittsburgh, PA (August 4, 1997). 
20. Dharmarajan, R.: Bayer Corporation. Pittsburgh, PA, to S. Levine, personal communication (January, 2002).

21. Spence, M.: Dow Chemical Company. Midland, MI, to S. Levine, personal communication (February 15, 2002).

22. Bello, D.; Streicher, R.P.; Woskie, S.R.: Evaluation of the NIOSH Draft Method 5525 for Determination of the Total Reactive Isocyanate Group (TRIG) in Air in Auto Body Repair Shops. Manuscript in preparation for publication. R.P. Streicher, NIOSH, Cincinnati, $\mathrm{OH}$, to S. Levine, personal communication (January, 2002).

23. Bello, D.; Woskie, S.R.; Streicher, R.P.; et al.: Field Comparison of Impingers and Treated Filters for Sampling of Total Aliphatic Isocyanates Using the MAP Method. Manuscript in preparation for publication. R.P. Streicher, NIOSH, Cincinnati, OH, to S. Levine, personal communication (January, 2002).

24. Rando, R.J.; Poovey, H.G.; Mokadam, D.: Evaluation of the ISOCHEK Sampler for Measurement of Monomers and Prepolymers of TDI and MDI, Project 155, Reference 11415. International Isocyanate Institute, Inc., Manchester, UK (2001).

25. NIOSH Method 5521: Isocyanates 1989. In: NIOSH Manual of Analytical Methods, 3rd ed., P.M. Eller, ed. (1984) with supplements (1985, 1987, 1989). NIOSH, Cincinnati, OH (May 15, 1989).

26. Organic Isocyanates in Air-MDHS Method 25/3. U.K. Health and Safety Laboratory, Sheffield, UK (1999).

27. Bagon, D.; Warwick, C.; Brown, R.: Evaluation of Total Isocyanate-in-Air Method Using 1-(2-Methoxyphenyl)Piperazine and HPLC. Am Indus Hyg Assoc J 45(1):39-43 (1984).

28. Parekh, P.: Huntsman Polyurethane Company. Cherry Hill, NJ, to S. Levine, personal communication (January 25, 2002).

29. ISO Method 16702:2001: Workplace Air Quality-Determination of Total Isocyanate Groups in Air Using 2-(1-Methoxyphenyl)Piperazine and Liquid Chromatography. International Organization for Standardization, Geneva (2001).

30. NIOSH Method 141: 2,4-TDI in Air. In: NIOSH Manual of Analytical Methods. NIOSH, Cincinnati, OH (1977).

31. Rando, R.J.; Hammand, Y.Y.: Modified Marcali Method for the Determination of Total TDI in Air. Am Indus Hyg Assoc J 46:206210 (1985).

32. Method 837: Determination of 2,4-TDI in Air. In: Methods of Air Sampling and Analysis, 3rd ed., J. Lodge, ed., Intersociety Committee APCA, ACS, AIChE, APWA, ASCE, ASME, AOAC, HPS, ISA, pp. 697-700. Lewis Publishers, Inc., Chelsea, MI (1989).

33. NIOSH Method 2535: 2,4-TDI. 1987. In: NIOSH Manual of Analytical Methods, P.M. Eller, ed., 3rd ed., with supplements (1985, 1987). NIOSH, Cincinnati, OH (1984).

34. OSHA Method 18: Diisocyanates. OSHA Analytical Laboratory, Organic Methods Development Branch, Salt Lake City, UT (1980).

35. Bayer CIHL Method No: 1.4.4. Revision 4. Bayer Corporation, Pittsburgh, PA (February 4, 1997).

36. NIOSH Method 5505: Isocyanate Group. In: NIOSH Manual of Analytical Methods, 3rd ed., P.M. Eller, ed., with supplements (1985, 1987). NIOSH, Cincinnati, OH (1984).

37. Seymour, M.; O'Connor, P.F.; Teass, A.: Investigation of the Inaccuracy of NIOSH Method 5505 for Estimating the Concentration of Isocyanate in Air. Appl Occup Environ Hyg 5(2):115-122 (1990).

38. Tinnergerg, H.; Karlsson, D.; Dalene, M.; et al.: Determination of TDI in Air Using Di-n-Butylamine and 9-N-Methylaminomethyl-
Anthracene as Derivatizing Reagents. J Liq Chrom Rel Technol 20:2207-2219 (1997).

39. Dharmarajan, V.: Evaluation of Personal Continuous Paper-Tape Monitors for TDI. Am Indus Hyg Assoc J 57:68-71 (1996).

40. Booth, K.S.; Dharmarajan, V.; Lingg, R.D.; et al.: State-ofthe-Art Monitoring and Analysis for Airborne Isocyanates. In: Polyurethane-Marketing and Technology-Partners in Progress. Proceedings of the SPI 28th Annual Technical/Marketing Conference Society of the Plastics Industry, San Antonio, TX (1984).

41. Dharmarajan, V.; Rando, R.: Critical Evaluation of Continuous Monitors for Toluene Diisocyanate. Am Indus Hyg Assoc J 41:869-878 (1980).

42. Rando, R.; DuVoisin, P.; Abdel-Kader, H.; et al.: A Sequential Tape Monitor for Toluene Diisocyanate. Am Indus Hyg Assoc J 48:574-579 (1987).

43. Groves, J.; Brown, R.; Szikora, J.; et al.: A Laboratory Study of Impinger Efficiencies and a Comparison of the Marcali Method, MDHS 25, and Paper Tape Monitors for the Measurement of 2,4 and 2,6 Toluene Diisocyanate. Health and Safety Executive/Research and Laboratory Services Division, Edgeware, London, UK (1985).

44. Milsom, E.; Groves, J.A.: Laboratory Evaluation of a Pumped Sampling Device for the On-Site Measurement of TDI. Report IR/L/SP/91/02 or project R48.50. HSE, London, UK (1991).

45. Walker, R.; Pinches, M.: Chemical Interference Effects in the Measurements of Atmospheric Toluene Diisocyanate Concentrations When Sampling with an Impregnated Paper Tape. Am Indus Hyg Assoc J 42:392-397 (1981).

46. Miller, J.; Mueller, F.: Evaluation of a Continuous Instrumental Method for Determination of Isocyanates. Am Indus Hyg Assoc J 35:477-483 (1975).

47. Coyne, L.B.: An Evaluation of TDI Monitoring Instruments. Presented at the American Industrial Hygiene Conference. Montreal, Canada (1987).

48. Dharmarajan, V.; Booth, K.S.: Evaluation of Paper-Tape Monitors in Laboratory-Generated TDI Atmospheres at Various $\mathrm{Hu}-$ midities and Concentrations. Poster presented at the Polyurethanes EXPO’96, Las Vegas, NV (Oct., 1996).

49. Nakaaki, K.: Comparative Study of HPLC Method and TDI Tape Monitor Method (Isologger): Field Survey in Japan. Project 164, Reference 11411. International Isocyanate Institute, Inc., Manchester, UK (2001).

50. Accuracy, Reliability and Alarms of Direct Reading Instruments for Diisocyanates-The Assembly Plant Experience. James D’Arcy, General Motors Research Laboratories, Warren, MI, to S. Levine, personal communication (November, 2001).

51. Yamaguchi, T. Zellweger Analytics, Chicago (Files detailing instrument performance metrics) to S. Levine, personal communication (February 4, 2002).

52. Safeair System Validation Report-TDI, Publication KM4-23B.00898. K\&M Environmental, Virginia Beach, VA (August, 1998).

53. Validated TDI Diffusion Monitor: Meeting the Challenge of Personnel Monitoring. K. Chapman, Polyurethanes Conference 2000. (Location and date not cited).

54. Workplace Analysis Scheme for Proficiency. Quality Assessment Scheme for the Analysis of Occupational Hygiene Samples. The Health and Safety Laboratory, Sheffield, UK.

55. Industrial Hygiene Laboratory Accreditation Program (IHLAP). American Industrial Hygiene Association, Fairfax, VA (2002). 